\title{
Construction and Practice of Experimental Demonstration Center for Agricultural Electrification and Automation
}

\author{
Hua Liu \\ College of Engineering and Technology \\ Tianjin Agricultural University \\ Tianjin, China \\ 41599386@qq.com
}

\author{
Weiyu Zhang(Corresponding Auther)* \\ College of Engineering and Technology \\ Tianjin Agricultural University \\ Tianjin, China \\ 525156985@qq.com
}

\author{
Chuanlin Li \\ College of Engineering and Technology \\ Tianjin Agricultural University \\ Tianjin, China \\ 361473215@qq.com \\ Yu Shi \\ College of Engineering and Technology \\ Tianjin Agricultural University \\ Tianjin, China \\ syych83@tjau.edu.cn
}

\begin{abstract}
Combined with the practice and construction of Agricultural Electrical Engineering and Automation Experimental Center in Tianjin Agricultural University, this paper elaborates on the position, concept and construction target of the experimental teaching center. A. Policy support, instrument \&instrument, 'project platform' operation management mode, information platform, experimental teaching system and content are introduced. The features of center are also comprehensively summarized. The center can establish experimental teaching platform, Personnel promotion, etc., improve the talent cultivating mode and improve comprehensive and innovative ability of students. Construction of the center has attained remarkable results, and built an excellent mode for demonstration and expanded its influence.
\end{abstract}

Keywords-Experimental Demonstration Center, Teaching Platform, Engineering Education, Innovative Ability

\section{INTRODUCTION}

Tianjin Agricultural University, Experimental Teaching Demonstration Center for Agricultural Electrification and Automation, approved in December 2012 in Tianjin experimental teaching demonstration center construction unit. The experiment with the development of the school and the center, to carry out teaching, research and social services working in the field of agricultural electrification and automation technology, detection and control of agricultural engineering, agricultural information[1].

The Centre has a middle-aged teachers as the backbone, the combination of high-quality part-time teachers in Annex 1, in which 37 full-time test team has the title of senior teachers and Ph.D. accounted for more than 59\% of the total number of experimental technique team, wherein the age of 35-45 years old accounted for between $72 \%$ number of senior positions, a high level of teaching the young team to ensure the smooth and continuously push forward the reform of teaching experimental teaching.

In the last three years, school invested 6.86 million yuan for the purchase of equipment test center. Starting in 2014, the Centre for professional integration laboratory is built "wall cabinets - Test Stands - computer" general laboratory Trinity 7 , in terms of time - space and laboratory utilization of reform for the Central Laboratory .

\section{CONSTRUCTION OF THE EXPERIMENT PlATFORM}

Center since its establishment, under various expenditures funded, acquires equipment 401 sets. The rate of turnover has reached more than $30 \%$, including 9 sets of large equipment, more than 10 million yuan, mainly fluorescence elemental analyzer, atomic absorption spectrometer, gas chromatograph, vector network analyzer. The facilities of the center cover curriculum categories, fully capable of electrical and automation, environmental and energy engineering related majors in the basic experiment, comprehensive experiment, professional design experiments teaching, meet the students the basic experimental skills and innovation ability training. Outside the center of the 24 internship bases for students make a good condition to promote their abilities[2].

\section{A. Policy Support}

School has always attached importance to experimental teaching, from the aspects of system, policy, manpower and material resources to ensure that the work of the experimental teaching status, information of the leadership of attention to education, the standard of the teaching system, policy tilt teaching, scientific research to promote teaching, teaching services, forming priority teaching good atmosphere[3]. 


\section{B. Configuration of Instrument and Equipment}

Demonstration center since its establishment, under various expenditures funded, acquired equipment 401 sets, turnover rate has reached more than 30\%, including 9 sets of large equipment, more than 10 million yuan, mainly fluorescence elemental analyzer, atomic absorption spectrometer, gas chromatograph, vector network analyzer. The facilities of the center cover curriculum categories, fully capable of electrical and automation, environmental and energy engineering related majors in the basic experiment, comprehensive experiment, professional design experiments teaching, meet the students the basic experimental skills and innovation ability training[4].

\section{Enhance the Teaching Team}

Nearly 3 years center through the introduction and internal training combining way, both to ensure the experimental teachers of the stability, guarantee the teaching task successfully completed, and timely supplement in experimental teaching has rich experience of senior personnel, keep the rational flow of personnel of experiment teaching team, promote the input and exploration of experimental teaching of advanced concepts, provide a guarantee to keep the experimental teaching demonstration center characteristics and the demonstration of radiation effects[5]. The main measures taken are as shown in figure 1:

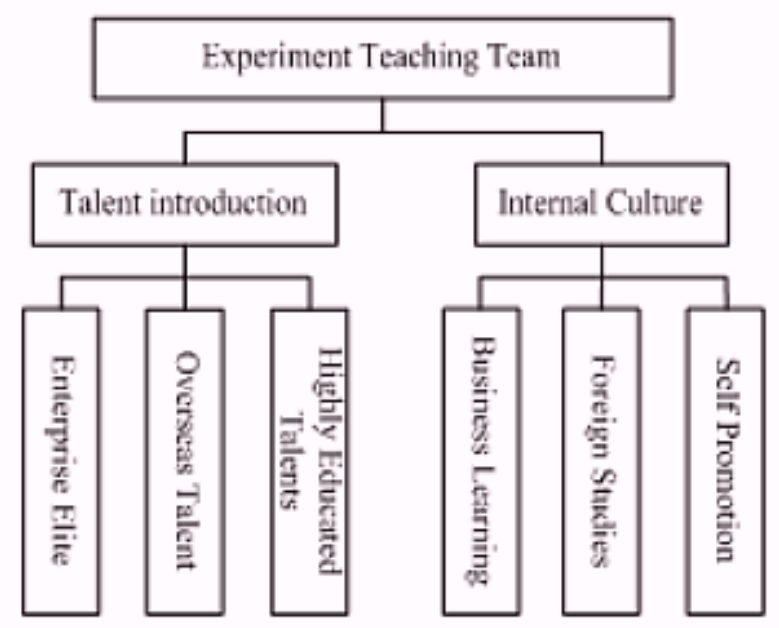

Fig. 1. Experiment Teaching Team for Agricultural Electrification and Automation Experiment Teaching Demonstration Center

It is to combine discipline construction, stabilize the talent troop and improve the teaching level. Making full use of the school's multi discipline advantage, relying on the discipline of the master's point, the basic realization of the "center construction and discipline construction combination" planning principles, the formation of the discipline construction and the center of the construction of the benign interaction. Discipline construction attracted young teachers into the center of the construction and development of the cause, so that they get in to stay ", lived a number of urgently, young backbone; discipline construction ensures the center construction to keep up with the development of advanced technology at home and abroad with a stable mechanism, expand the new experimental techniques for field, updating the teaching contents, to build a platform for innovation. Construction of subject construction.

\section{Experiment \& Training Project Platform}

As shown in Fig.2, it is the construction for the experiment \& training project platform for the experimental teaching demonstration center[6].

Center in the experimental project platform to open a basic experiment, comprehensive innovative experimental project. On this basis, students complete the basic experiment and comprehensive experiment and learn to spare, the students can according to their own circumstances, participate in contests, teachers' scientific research and enterprise project.

\section{E. Information Platform}

Since 3 years, center organize relevant personnel to participate the meeting held in Beijing, Tianjin, a national experimental teaching demonstration center display exchange opportunity to consult the more than a dozen demonstration unit of the information center building situation, and the unit website construction of Southeast University, Dalian University of technology, China Agricultural University, Ocean University of China and Sichuan University. Information construction in the center of the school investment funds. Update and improve the experimental teaching demonstration center of experimental teaching resources information management subsystem, experimental teaching demonstration center of experimental teaching resources information distribution subsystem and experimental teaching demonstration center service subsystem.

Under the support of the information platform, do the unified management, reasonable allocation of shared use of multi-class, and open to the whole school teachers and students, the conventional instruments and equipment utilization rate reached $99.7 \%$ and applied to the use of largescale instruments and equipment with experimental teaching of undergraduate students rate reached more than $90 \%$, than before the establishment of the center has been greatly improve. 


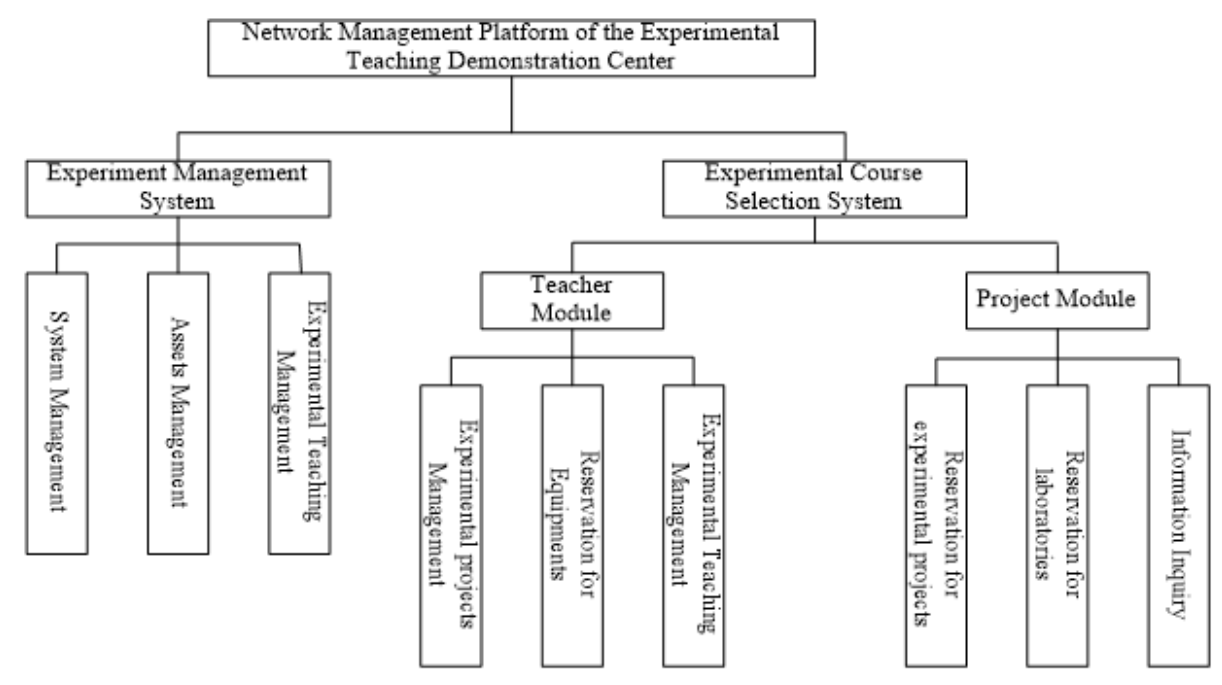

Fig. 2. Experiment \& training project platform for the experimental teaching demonstration center

\section{F. Management Mode}

Experimental teaching center set up the center office. The office is to assist the center director to carry out a full range of daily management of the center's laboratory construction, experimental teaching, personnel team, and information data and so on.

At the same time, it set up the center of large instrument management group, safety and health group, information data group, the purchase and use, maintenance, opening of the center of the large and medium-sized instruments, the implementation of supervision and inspection.

\section{SIGNIFICANT EXPERIMENT TEACHING EFFECT}

In recent three years, the center approved research projects 33 , teaching reform project 12 , reporting and approved patents and software copyright 33, published 124 papers, 35 educational reform papers.

"Hands and brain, comprehensive training, tamp foundation, innovation", experimental center will continue to rely on the College of engineering and technology, a high level of scientific research team, the teaching theory, combined with the scientific research and experiment and practice teaching organically, and strive to build experimental center of training undergraduate students the basic experimental skills, creative thinking and comprehensive scientific literacy of the base. Since 2013, with 24 enterprises and institutions to establish a combination of the nature of the practice and training base.

New system of experimental teaching and the students' innovation consciousness and innovation ability has been significantly improved, since 2013, the experimental center of undergraduates take the National College Students' innovative training project 28, Tianjin innovation project 32, University
Innovation Project 26, receive funding 44.6 million yuan, in have a big project, 6 to obtain excellent project; undergraduate in domestic and foreign academic journals: 78 papers, students participate in 20 patents; students to participate in various levels to the class discipline competition winning 58; through the innovative activities of students, 3 people get Tianjin students innovation scholarship.

\section{CONCLUSIONS}

\section{A. "Characteristic" Philosophy}

Agricultural Electrification and Automation Experimental Teaching Center adhere to the "people-oriented, unique teaching, laying equal stress on both theoretical teaching and practical teaching, experimental skill training and the training of creative thinking", simultaneously teaching idea, promote students' knowledge, ability and quality comprehensive sustainable coordinated development. The characteristics of teaching "is according to the Tianjin Agricultural College" training service of coastal city type modern agriculture, with creation consciousness, the spirit of innovation, entrepreneurial ability and basic quality of 'three radicals' high-quality applied talents " guiding ideology and orientation of running a school record, combined with the subject and resource advantage, based on the needs of the economic development of Tianjin modern agriculture, to the field of precision agriculture in rural power system and automation technology, agricultural products detection, agricultural mechanization and automation technology and agricultural information for both research and teaching focus on the direction of the. To emphasize that under the constructivism theory to guide, teacher as a guide, take the student as the center, in the theory of learning based, to cultivate students' practical ability and innovative ability as the goal, focus on students' practical skills, scientific thinking, team consciousness and innovative spirit training, and constantly 
improve the students' innovation ability and the ability of sustainable development.

Agricultural Electrification and Automation of Experimental Teaching Center adhere to the "people-oriented, features teaching, practice teaching and theory teaching both experimental skills and creative thinking training " teaching philosophy, to promote students' knowledge, ability, quality and overall sustainable development. "Features Teaching" is based on Tianjin Agricultural College "Training Services Coastal Urban Modern Agriculture with creating awareness, the spirit of innovation, entrepreneurship and the basic quality of the 'three hit a group' Qualified Personnel" guiding ideology and school Location combining disciplines and resources, for Tianjin modern agriculture economic development needs of rural power systems and automation technology, testing of agricultural products, agricultural mechanization equipment, automation technology, information technology and other agricultural areas of precision agriculture research and teaching focus direction. He emphasized in the build theory pointing, teacher guide, student-centered, based on the theoretical study on cultivating students 'practical and innovative ability as the goal, focus on students' practical skills, scientific thinking, and teamwork and innovation culture and constantly improve the students ability to innovate and sustainable development.

\section{B. Integrating the Advantages of Enterprise Resources, the Sustainable Development of School Enterprise Combination}

Modern agriculture in Tianjin city of electrification and automation of demand oriented setting experimental training project, in strengthening the basic experiment, at the same time, to create a line with Tianjin agricultural electrification and automation engineering environment, let the students understand the modern agricultural production mode and process flow, so as to master and improve their engineering skills.

After 3 years of construction, the center has gradually formed the class tutor responsible system of project teaching method, arouse and improve the students' interest in learning and academic competitions normalization and improve the students' ability of innovation, promote the theory teaching, experiment teaching, innovation practice of scientific research and teacher of "zero distance" contact.

\section{Comprehensive Experimental Base}

The basis of the original in recent years, have been purchased a large number of experimental equipment, especially the American company's PE detection equipment, and gradually formed a PE spectroscopy equipment company in Tianjin area gathered, enhances the teaching experiment equipment of integrated, comprehensive; and integration of the general laboratory for experiment and scientific research to provide a convenient time and space. Center for the whole school, provides the high quality resources of experimental teaching for undergraduate teaching, but also for graduate teaching, training, research and social services; laboratory fully open to undergraduates, students as soon as possible to experience autonomous learning and receiving conditions and resources from the scientific research training, improve the platform resources use efficiency, to achieve a high degree of sharing resources in a comprehensive and open.

\section{ACKNOWLEDGMENT}

This work was financially supported by the Educational Reform project for the Experimental Teaching Center of Tianjin Agricultural University (2015SY024 and 2015SY16).

\section{REFERENCES}

[1] Wang Lili, Ai Xin, Song Jinpeng. Construction and Practice of National Experimental Teaching Demonstration Cener for Electrical Engineering [J]. Experimental Technology and Management. 2014,12(31):124-127.

[2] Huang Jiu Jiu, Wang Sheng bin, Gong Hui. Modern biotechnology experimental teaching center construction practice and thinking [J]. Laboratory science, 2014,02:180-183+187.

[3] Wang Ligui. The construction and practice of the experimental teaching demonstration center of national economic management [J]. laboratory research and exploration, 2011,02:98-100+148.

[4] Fan Gongguang, Liu Yongping, Xie Jun. National experimental teaching demonstration center of experimental teaching system exploration and practice [J]. Experimental technology and management, 2013,02:134136.

[5] Yu Zhongan, Zhang Zhenli. To strengthen the construction of experimental teaching demonstration center to improve the quality of practice teaching $[\mathrm{J}]$. Journal of Jiangxi University of Science and Technology, 2010,06:49-52.

[6] Li Yudong, Liu Jingyan, Yan Youyun. Enhancing Practical Teaching Reform and Cultivating Outstanding Engineers Depending on Experimental Teaching Demonstraion Center[J]. Research and Explore in Laboratory, 2012,3(31):105-108. 